\title{
Mitochondrial dynamics and biogenesis indicators may serve as potential biomarkers for diagnosis of myasthenia gravis
}

\author{
LANQI LI ${ }^{1,2}$, DONGHONG CAI ${ }^{1,2}$, HUIYA ZHONG ${ }^{1,2}$, FENGBIN LIU $^{3}$, QILONG JIANG $^{3}$, \\ JIAN LIANG ${ }^{4}$, PEIWU LI ${ }^{3}$, YAFANG SONG ${ }^{1,2}$, AIDONG JI $^{5}$, WEI JIAO ${ }^{1,2}$, JINGWEI SONG $^{1,2}$, \\ JINQIU LI ${ }^{1,2}$, ZHIWEI CHEN ${ }^{1,2}$, QING LI $^{1,2}$ and LINGLING KE ${ }^{1,2}$
}

\begin{abstract}
${ }^{1}$ Science and Technology Innovation Center; ${ }^{2}$ Institute of Pi-Wei, Guangzhou University of Chinese Medicine;
${ }^{3}$ Department of Gastrosplenic Diseases, The First Affiliated Hospital of Guangzhou University of Chinese Medicine,

Guangzhou, Guangdong 510405; ${ }^{4}$ Guangdong Provincial Key Laboratory of New Drug Development and Research of Chinese Medicine, Mathematical Engineering Academy of Chinese Medicine; ${ }^{5}$ Clinical Medical College of Acupuncture, Moxibustion and Rehabilitation, Guangzhou University of Chinese Medicine, Guangzhou, Guangdong 510006, P.R. China
\end{abstract}

Received October 27, 2021; Accepted February 10, 2022

DOI: $10.3892 / \mathrm{etm} .2022 .11236$

\begin{abstract}
Due to challenges in diagnosing myasthenia gravis (MG), identifying novel diagnostic biomarkers for this disease is essential. Mitochondria are key organelles that regulate multiple physiological functions, such as energy production, cell proliferation and cell death. In the present study, Mfn1/2, Opa1, Drp1, Fis1, AMPK, PGC-1 $\alpha$, NRF-1 and TFAM were compared between patients with MG and healthy subjects to identify potential diagnostic biomarkers for MG. Blood samples were collected from 50 patients with MG and 50 healthy subjects. The participants' demographic information and routine blood test results were recorded. Mitochondrial dynamics were evaluated and levels of Mfn1/2, Opa1, Drp1, Fis1, AMPK, PGC-1 $\alpha$, NRF-1 and TFAM were determined in peripheral blood mononuclear cells using western blotting and reverse transcription-quantitative PCR, respectively. Receiver operating characteristic curve analysis was used to evaluate the diagnostic accuracy of these indicators. The areas under
\end{abstract}

Correspondence to:Professor Yafang Song, Science and Technology Innovation Center, Guangzhou University of Chinese Medicine, 12 Baiyun District Airport Road, Guangzhou, Guangdong 510405, P.R. China

E-mail: stephanie237@163.com

Abbreviations: Ab, antibody; AMPK, AMP-activated protein kinase; Drp, dynamin-related protein; Fis, fission; Mfn, mitofusion; MG, myasthenia gravis; mtDNA, mitochondrial DNA; NRF-1, nuclear respiratory factor-1; OMM, outer mitochondrial membrane; Opa 1, optic atrophy type 1; PGC-1 $\alpha$, peroxisome proliferator-activated receptor- $\gamma$ co-activator- $1 \alpha$; ROC, receiver operating characteristic; TFAM, mitochondrial transcription factor A

Key words: myasthenia gravis, mitochondrial dynamics, biogenesis, peripheral blood mononuclear cells, biomarkers the curve values of Mfn1/2, Opa1, Drp1, Fis1,AMPK, PGC-1 $\alpha$, NRF-1 and TFAM were 0.5408-0.8696. Compared with control subjects, mRNA expression levels of Mfn1/2, Opa1, AMPK, PGC- $1 \alpha$, NRF- 1 and TFAM were lower, while those of Drp1 and Fis1 were higher in patients with MG. The protein expression levels of all these molecules were lower in patients with MG than in control subjects. These results suggested that mitochondrial dynamics and biogenesis indicators may be diagnostic biomarkers for MG.

\section{Introduction}

Myasthenia gravis (MG) is an autoimmune disease characterized by dysfunctional transmission of nerve impulses to muscles (1), which impedes eyelid movement, facial expression, chewing, talking, swallowing and breathing. The global incidence of MG is 1.7-21.3/1,000,000 individuals per year (2). Although studies have shown that the mortality rate of MG is currently lower than two decades ago $(3,4)$, MG still impacts the quality of life of patients. In current clinical practice, MG diagnosis is dependent on disease stage and involves clinical examination based on serum autoantibody detection. The specific diagnostic tools evaluate levels of anti-acetylcholine receptor (AChR) and anti-muscle-specific tyrosine kinase (MuSK) Abs (5). However, 10\% of patients with MG test negative for both anti-AChR and anti-MuSK Abs (6). Therefore, developing novel methods to diagnose MG is vital.

Mitochondria are abundant in muscle cells and provide most of the energy required to maintain daily activity of the human body (7). They are key for energy production and cell proliferation and death (8). Mitochondrial function is regulated and depends on structure, dynamics and biogenesis (9). Mitochondria serve a key role in improving the metabolic quality and plasticity of skeletal muscles by maintaining biogenesis, dynamics and autophagy/mitophagy (10).

Mitofusion 1 (Mfn1), Mfn2, optic atrophy type 1 (Opa1), dynamin-related protein 1 (Drpl) and fission 1 (Fis1) are key factors associated with mitochondrial fusion and fission (11). 
Mfn1 and Mfn2 are located in the outer mitochondrial membrane (OMM) and regulate mitochondrial fusion. Opa1 is a member of the dynamin family of mechanoenzymes that are localized in the inner MM, where they regulate mitochondrial fusion (12).

Previous studies have indicated that regulation of Mfn2 to restore mitochondrial homeostasis inhibits development of diabetic cardiomyopathy $(13,14)$. Drp1 dissociates in the cytosol, while Fis1 is anchored to the OMM. Specific signals induce transfer of Drp1 from the cytosol to the surface of the organelle where it interacts with Fis1 to complete organelle division $(12,15)$. This physiological process is known as mitochondrial dynamics (8).

AMP-activated protein kinase (AMPK) is an energy metabolism receptor that serves an important role in maintaining balanced energy metabolism in cells (16). Peroxisome proliferator-activated receptor- $\gamma$ co-activator- $1 \alpha$ (PGC- $1 \alpha$ ) is the primary transcriptional regulator of mitochondrial biogenesis, respiration and oxidative phosphorylation (17). Nuclear respiratory factor-1 (NRF-1) is a nuclear transcription factor that stimulates expression of nuclear genes to enhance mitochondrial respiratory function. Mitochondrial transcription factor A (TFAM), a DNA-binding protein, controls mitochondrial metabolism and dysfunction by regulating the transcription of its genome and organizing mitochondrial DNA (mtDNA) (18). PGC-1 $\alpha$ activates TFAM by serving as a co-transcription factor of NRF-1, thereby regulating mitochondrial biogenesis (19).

Previous studies have shown that mitochondria serve a role in the development of rare neuromuscular diseases, such as Duchenne and Becker muscular dystrophy $(20,21)$. Mitochondrial dysfunction affects muscle function, leading to atrophy (22). Muscle weakness is the primary symptom of MG. Both muscle contraction and relaxation require mitochondria for energy supply; the dynamic balance and biogenesis of mitochondria are key for these processes (23). Therefore, it was hypothesized that mitochondrial dynamics and biogenesis factors may serve as diagnostic biomarkers for MG. The aim of the present study was to investigate the differences in mitochondrial dynamics and biogenesis between patients with MG and healthy individuals. Receiver operating characteristics (ROC) curves were plotted using reverse transcription-quantitative (RT-q)PCR data to evaluate the diagnostic value of mitochondria-associated genes, as well as to determine whether mitochondrial dynamics and biogenesis factors can serve as diagnostic biomarkers for MG.

\section{Materials and methods}

Participants. Patients with MG (19 males and 31 females) were enrolled from The First Affiliated Hospital of Guangzhou University of Chinese Medicine (Guangzhou, China) between August 2018 and February 2019. Samples from 50 healthy volunteers (25 males, 25 females) were also included in the present cross-sectional study. The following inclusion criteria were used: i) Age, $14-75$ years, ii) willing to participate in the study and iii) examination by personnel trained in diagnosis of MG class IIb (based on the clinical classification proposed by the MG Foundation of America) (24). The following exclusion criteria were used: i) Hormone, immunosuppression, plasmapheresis, or intravenous $\gamma$ globulin treatment during the previous 3 months, ii) serious infectious disease or subsequent complications (such as mental illness; cerebrovascular, heart or liver disease; kidney failure; or malignant tumor) and iii) participation in another clinical study in the past 3 months. All participants understood the experimental procedure and provided written informed consent. Written informed consent was obtained from the parents/guardians of all participants $<18$ years old. All procedures involving human subjects were approved by the Academic Ethics Committee of The First Affiliated Hospital of Guangzhou University of Chinese Medicine.

Isolation of human peripheral blood mononuclear cells (PBMCs). Peripheral blood samples $(5 \mathrm{ml})$ were collected in an anticoagulant tube. Within $1 \mathrm{~h}$ of sample collection, PBMCs were isolated using Ficoll-Paque ${ }^{\mathrm{TM}}$ gradient (GE Healthcare). An equal volume of phosphate buffer was added to dilute the blood sample, which was added to the Ficoll-Paque separating solution and centrifuged at $600 \mathrm{x} \mathrm{g}$ for $20 \mathrm{~min}$ at $25^{\circ} \mathrm{C}$. The samples were separated into four layers. PBMCs were collected from the second layer (mononuclear cells). PBMCs were washed three times with phosphate-buffered saline and centrifuged $\left(600 \times \mathrm{g}, 10 \mathrm{~min}, 4^{\circ} \mathrm{C}\right)$, and then stored at $-80^{\circ} \mathrm{C}$ (Fig. 1).

Total RNA extraction. Total RNA was isolated from PBMCs using TRIzol ${ }^{\circledR}$ reagent, according to the manufacturer's instructions (Invitrogen; Thermo Fisher Scientific, Inc.). TRIzol reagent $(1 \mathrm{ml})$ and chloroform $(0.2 \mathrm{ml})$ were added to each sample tube. The tubes were mixed for $15 \mathrm{sec}$, allowed to stand for $3 \mathrm{~min}$ and centrifuged at $600 \mathrm{x} \mathrm{g}$ for $15 \mathrm{~min}$ at $4^{\circ} \mathrm{C}$. The upper aqueous phase was carefully aspirated and transferred to a new tube. An equal volume of isopropanol was added and samples were mixed at $25^{\circ} \mathrm{C}$ for $20 \mathrm{~min}$. The samples were centrifuged at $13,000 \mathrm{x} \mathrm{g}$ for $10 \mathrm{~min}$ at $4^{\circ} \mathrm{C}$ to precipitate the RNA and the supernatant was removed. The sediment was washed with $1 \mathrm{ml}$ pre-cooled $75 \%$ ethanol and centrifuged again $\left(600 \times \mathrm{g}, 5 \mathrm{~min}, 4^{\circ} \mathrm{C}\right)$. After discarding the supernatant, the RNA pellet was dissolved in $30 \mu \mathrm{l}$ diethyl pyrocarbonate-treated water and stored at $-80^{\circ} \mathrm{C}$.

$R T$-qPCR evaluation of target $m R N A$ expression in PBMCs. Total RNA was extracted from PBMCs using TRIzol ${ }^{\circledR}$ reagent as aforementioned and reverse-transcribed to cDNA using PrimeScript $^{\mathrm{TM}}$ RT Master Mix (cat. no. RR036Q; Takara Bio, Inc.) according to the manufacturer's instructions. The mRNA expression levels were measured using SYBR Green Master Mix (cat. no. RR036A; Takara Bio, Inc.) on a CFX96 Real-Time PCR System (Bio-Rad Laboratories, Inc.), according to the manufacturer's instructions, using the following thermocycling conditions: Initial denaturation at $95^{\circ} \mathrm{C}$ for $20 \mathrm{sec}$, followed by 40 cycles of $95^{\circ} \mathrm{C}$ for $10 \mathrm{sec}$, $60^{\circ} \mathrm{C}$ for $30 \mathrm{sec}$ and $70^{\circ} \mathrm{C}$ for $1 \mathrm{sec}$. The target gene sequences were obtained from GenBank (https://www.ncbi.nlm.nih. gov/genbank/) and primer sequences for all target genes are listed in Table I. Relative expression was determined using the $2^{-\Delta \Delta \mathrm{Cq}}$ method (25). GAPDH was used as the reference gene.

Western blotting evaluation of protein expression in PBMCs. PBMCs were lysed on ice with RIPA lysis buffer 
Table I. Primers used for reverse transcription-quantitative PCR.

\begin{tabular}{lll}
\hline Gene & \multicolumn{1}{c}{ Forward primer, 5'-3' } & \multicolumn{1}{c}{ Reverse primer, 5'-3' } \\
\hline Mfn1 & ATGTAACGGACGCCAATC & ATCTTTAGCTTCTACTCCCACT \\
Mfn2 & TGCAGGTGTAAGGGACGATT & GAGGCTCTGCAAATGGGATG \\
Opa1 & TGTCCTCCGCAAAGTCAT & TGCTTGGGAGACCCTACA \\
Drp1 & CAAAGCAGTTTGCCTGTGGA & TCTTGGAGGACTATGGCAGC \\
Fis1 & CCAGGTAGAAGACGTAATCCC & GTCCAAGAGCACGCAGTTT \\
AMPK & TTGAAACCTGAAAATGTCCTGCT & GGTGAGCCACAACTTGTTCTT \\
PGC-1 $\alpha$ & TCAGTCCTCACTGGTGGACA & TGCTTCGTCGTCAAAAACAG \\
NRF-1 & GGTGCAGCACCTTTGGAGAA & CCAGAGCAGACTCCAGGTCTTC \\
TFAM & CACATTTTCCACCTGGTGAT & CACTCCGCCCTATAAGCATC \\
\end{tabular}

Mfn, mitofusion; Opa, optic atrophy; Drp, dynamin-related protein; Fis, fission; AMPK, AMP-activated protein kinase; PGC, peroxisome proliferator-activated receptor- $\gamma$ co-activator; NRF, nuclear respiratory factor; TFAM, mitochondrial transcription factor A.

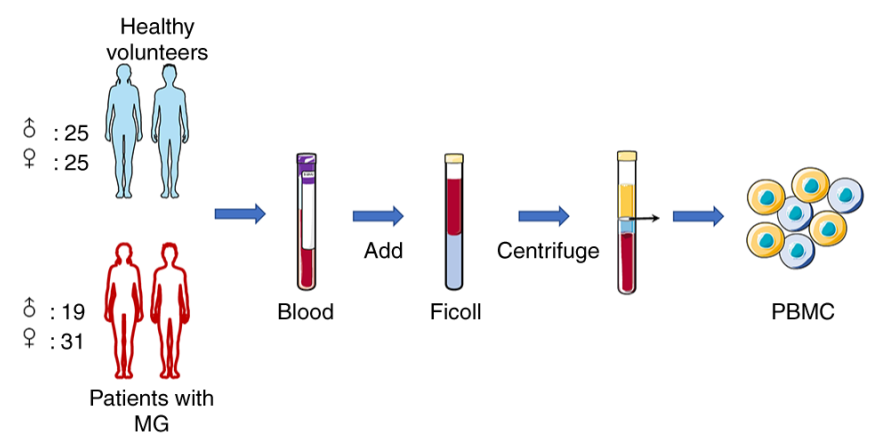

Figure 1. Isolation of human PBMCs. PBMC, peripheral blood mononuclear cell; MG, myasthenia gravis.

(cat. no. 78510; Thermo Fisher Scientific, Inc.) and centrifuged at $13,000 \mathrm{x}$ g for $15 \mathrm{~min}$ at $4^{\circ} \mathrm{C}$, and then supernatant was collected. Protein concentration was determined using the bicinchoninic acid method. An equal amount $(30 \mu \mathrm{g}, 20 \mu \mathrm{l})$ of protein was loaded/lane and samples were separated by $12 \%$ sodium dodecyl sulfate-polyacrylamide gel electrophoresis. Samples were transferred onto polyvinylidene difluoride membranes using a Trans-Blot Turbo Transfer System (Bio-Rad Laboratories, Inc.) and blocked at $25^{\circ} \mathrm{C}$ with $5 \%$ non-fat powdered milk for $1.5 \mathrm{~h}$. The membranes were incubated overnight at $4^{\circ} \mathrm{C}$ with the following primary antibodies (all Abcam): Anti-Mfn1 (cat. no. ab129154; rabbit; 1:2,000 in 5\% non-fat dry milk), anti-Mfn2 (cat. no. ab124773; rabbit; 1:1,000 in 5\% non-fat dry milk), anti-Opa1 (cat. no. ab42364; rabbit; 1:2,000 in 5\% BSA), anti-Drp1 (cat. no. ab219596; rabbit; 1:2,000 in 5\% BSA), anti-Fis1 (cat. no. ab229969; rabbit; 1:200 in 5\% non-fat dry milk), anti-AMPK (cat. no. ab80039; mouse; 1:1,000 in 5\% non-fat dry milk), anti-phosphorylated-AMPK (cat. no. ab133448; rabbit; $1: 1,000$ in 5\% non-fat dry milk), anti-PGC-1 $\alpha$ (cat. no. ab54481; rabbit; 1:1,000 in 5\% non-fat dry milk), anti-NRF-1 (cat. no. ab34682; rabbit; 1:1,000 in 5\% non-fat dry milk), anti-TFAM (cat. no. ab176558; rabbit; 1:1,000 in 5\% non-fat dry milk) and anti-GAPDH (cat. no. ab8245; mouse; 1:5,000 in 5\% non-fat dry milk). The membranes were then incubated with goat anti-rabbit (cat. no. ab7085; 1:3,000 in $5 \%$ non-fat dry milk) or goat anti-mouse (cat. no. ab7063; $1: 3,000$ in $5 \%$ non-fat dry milk) secondary antibodies for $90 \mathrm{~min}$ at $25^{\circ} \mathrm{C}$, then washed three times for 5 min each using Tris-buffered saline and polysorbate 20 (Beijing Solarbio Science \& Technology Co., Ltd.). Protein bands were visualized using Clarity ${ }^{\mathrm{TM}}$ Western ECL Substrate kit (Bio-Rad Laboratories, Inc.). The chemiluminescent signal was captured using a ChemiDoc ${ }^{\mathrm{TM}} \mathrm{XRS}+$ system and resulting bands were analyzed using Image Lab software version 3.0 (both Bio-Rad Laboratories, Inc.).

Statistical analysis. The sensitivity and specificity of variables for MG diagnosis were determined by ROC curve analysis using a non-parametric approach. The optimal cutoff values were selected based on those that minimized the sensitivity-specificity difference and maximized the discriminating power of the tests. All data were repeated three times and analyzed using SPSS 22.0 (IBM Corp.) and are expressed as the mean \pm standard deviation. Unpaired t-test was used to compare differences between control and $\mathrm{MG}$. $\mathrm{P}<0.05$ was considered to indicate a statistically significant difference.

\section{Results}

Participant information. A total of 50 healthy volunteers (25 males and 25 females; mean age, 38.92 \pm 14.76 years) were recruited for the control group and 50 patients with MG (19 males and 31 females; mean age, $42.58 \pm 13.76$ years) were recruited for the MG group. The clinical characteristics of participants are listed in Table II (raw data are shown in Table SI). A higher number of women than men were affected by MG, which was consistent with clinical reports $(26,27)$, and $66 \%$ of patients with MG were aged 30-60 years. According to Table II, the proportion of MG patients with a disease span of 0-6 years was $88 \%$. The patients exhibited dysphagia, chewing weakness, thymectomy and thyroid dysfunction (Table II). The hematology results of the patients with MG are shown in Table III. Routine blood examination values were all within normal ranges. The results provide comprehensive information on the participants. 
A

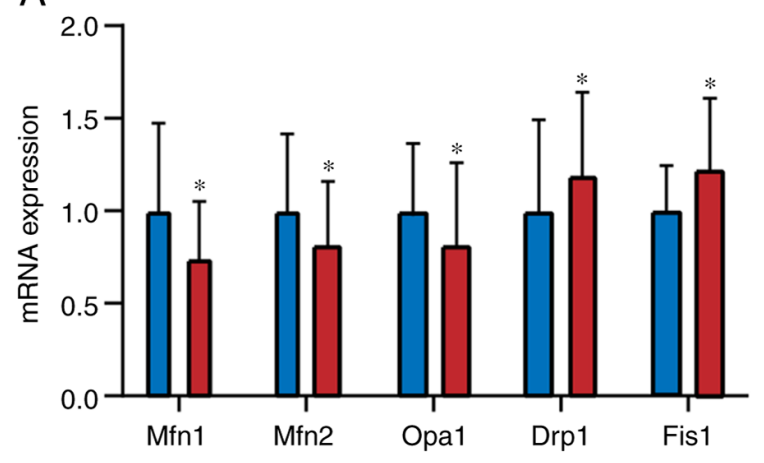

B

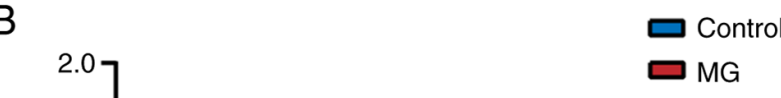

Figure 2. mRNA expression levels of mitochondrial fusion/fission-associated genes in peripheral blood mononuclear cells. (A) Mfn1/2, Opa 1, Fis 1, Drp 1, (B) AMPK, PGC-1 $\alpha$, NRF-1 and TFAM, mRNA expression levels in control and MG group. Data are presented as the mean \pm SD. ${ }^{*}<<0.05$, ${ }^{* *}$ P $<0.01$ vs. control. Mfn, mitofusion; Opa, optic atrophy; Drp, dynamin-related protein; Fis, fission; AMPK, AMP-activated protein kinase; PGC, peroxisome proliferatoractivated receptor- $\gamma$ co-activator; NRF, nuclear respiratory factor; TFAM, mitochondrial transcription factor A.

Table II. Clinical characteristics of patients and controls.

\begin{tabular}{lcc}
\hline Characteristic & MG $(\mathrm{n}=50)$ & Control $(\mathrm{n}=50)$ \\
\hline Sex & & \\
Male & 19 & 25 \\
Female & 31 & \\
Age, years & & 23 \\
14-29 & 9 & 6 \\
30-44 & 20 & 17 \\
$45-59$ & 16 & 4 \\
$\geq 60$ & 5 & - \\
Course of disease, months & & - \\
0-36 & 36 & - \\
$37-72$ & 8 & - \\
$73-108$ & 4 & - \\
$>108$ & 2 & - \\
Symptom (+) & & - \\
Neostigmine & 10 & - \\
AchR-Ab & 50 & - \\
MuSK-Ab & 44 & \\
Dysphagia & 30 & - \\
Chewing weakness & 25 & \\
Thymectomy & 11 & \\
Thyroid dysfunction & 28 & - \\
\hline
\end{tabular}

MG, myasthenia gravis; AchR, acetylcholine receptor; Ab, antibody; MuSK, muscle-specific tyrosine kinase.

mRNA expression of Mfn1, Mfn2, Opal, Drpl, Fis1, AMPK, $P G C-1 \alpha, N R F-1$ and TFAM measured by RT-qPCR. mRNA expression levels of Mfn1, Mfn2 and Opa1 were significantly lower in the $\mathrm{MG}$ than the control group $(\mathrm{P}<0.05$; Fig. 2; Table SII). By contrast, mRNA expression of Drp1 and Fis1 was higher in the MG group than the control $(\mathrm{P}<0.05)$. In addition, mRNA expression of AMPK, PGC-1 $\alpha$, NRF-1 and TFAM was significantly lower in the MG group than in the control $(\mathrm{P}<0.05)$. The results of $6 \mathrm{MG}$ patients who were
anti-AChR-negative are shown in Table SIII. According to the comparison of the results, there were differences in mRNA expression between control group and MG group patients (including antibody-negative patients).

Diagnostic value of Mfn1, Mfn2, Opal, Drpl, Fis1, AMPK, $P G C-1 \alpha, N R F-1$ and TFAM. To determine whether mitochondria-associated genes serve as diagnostic biomarkers for $\mathrm{MG}$, ROC curves were plotted using RT-qPCR data (Fig. 3). In ROC curve analysis (28), the area under the curve (AUC) quantifies the diagnostic potential of each candidate biomarker with a high AUC indicating a more accurate distinction between patients with MG and controls. AUCs were 0.8628 for PGC- $1 \alpha$, 0.8696 for NRF-1, 0.8072 for TFAM, 0.6488 for Mfn1, 0.6441 for Mfn2, 0.6212 for Opa1, 0.6468 for Drp1, 0.6604 for Fis1 and 0.5408 for AMPK (Fig. 3A-I). The results showed that mitochondrial energy metabolism (as measured by PCG-1 $\alpha$, NRF-1 and TFAM) had higher diagnostic value for MG.

Evaluation of Mfn1, Mfn2, Opa1, Drpl, Fis1, AMPK, PGC-1 $\alpha$, NRF-1 and TFAM expression by western blotting. Western blotting was performed to determine whether proteins were differentially expressed between control and MG groups. Mitochondrial dynamics-associated proteins (Mfn1, Mfn2, Opa1, Drp1 and Fis1) were expressed at significantly lower levels in PBMCs from patients with MG than in control subjects ( $\mathrm{P}<0.05$; Fig. 4). Mitochondrial biogenesis-associated proteins (AMPK, PGC-1 $\alpha$, NRF-1 and TFAM) were also expressed at lower levels in patients with $\mathrm{MG}$ than in control subjects $(\mathrm{P}<0.05$; Fig. 5). The results indicate that these mitochondrial markers may have diagnostic value for MG.

\section{Discussion}

Mitochondria are abundantly present in skeletal muscle fibrils, which require large amounts of ATP for contraction and diastolic movement (29). Mitochondria are semi-autonomous organelles that are key sites of tricarboxylic acid cycle reactions; their normal function determines whether skeletal muscles contract freely and flexibly (30). The accepted mechanism of skeletal muscle contraction (sliding filament theory) asserts that, when stimulated by neurotransmitters and in the presence 
Table III. Hematology results of patients with myasthenia gravis (mean $\pm \mathrm{SD}, \mathrm{n}=50$ ).

\begin{tabular}{lcccc}
\hline Variable & Male $(\mathrm{n}=19)$ & Normal male range & Female $(\mathrm{n}=31)$ & Normal female range \\
\hline $\mathrm{WBC}, 10^{3} / \mu \mathrm{l}$ & $8.59 \pm 4.63$ & $4.0-10.0$ & $9.94 \pm 3.13$ & $4.0-10.0$ \\
$\mathrm{RBC}, 10^{6} / \mu \mathrm{l}$ & $4.45 \pm 0.57$ & $4.0-5.5$ & $4.58 \pm 0.62$ & $3.5-5.0$ \\
$\mathrm{PLT}, 10^{3} / \mu \mathrm{l}$ & $227.11 \pm 60.22$ & $100.0-300.0$ & $229.78 \pm 41.86$ & $100.0-300.0$ \\
$\mathrm{Hb}, \mathrm{g} / 1$ & $130.89 \pm 19.18$ & $120.0-160.0$ & $130.42 \pm 18.06$ & $110.0-150.0$ \\
\hline
\end{tabular}

WBC, white blood cell; RBC, red blood cell; PLT, platelet; Hb, hemoglobin.

A

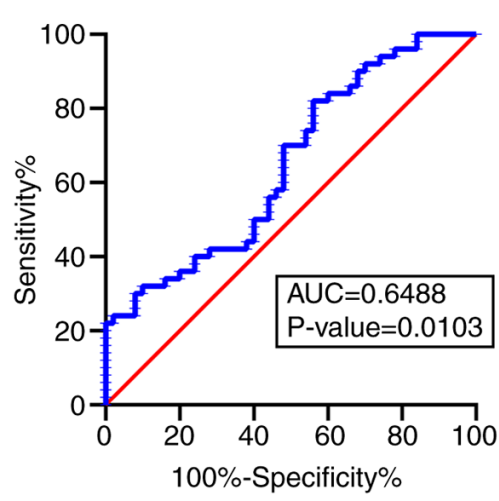

D

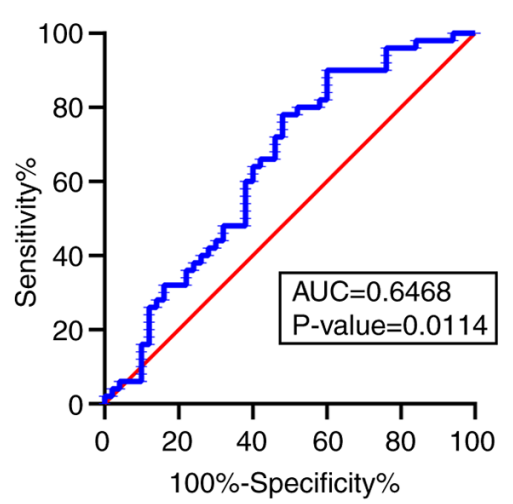

G

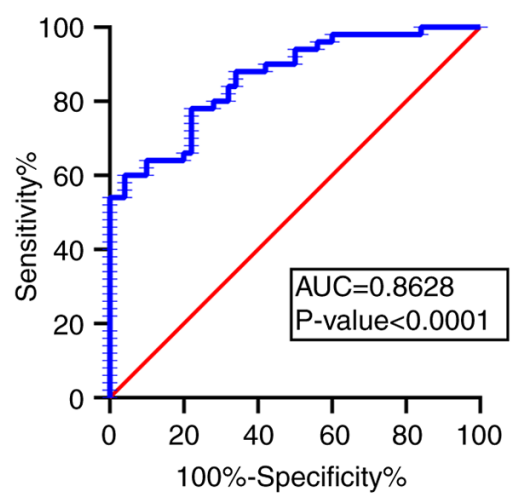

B

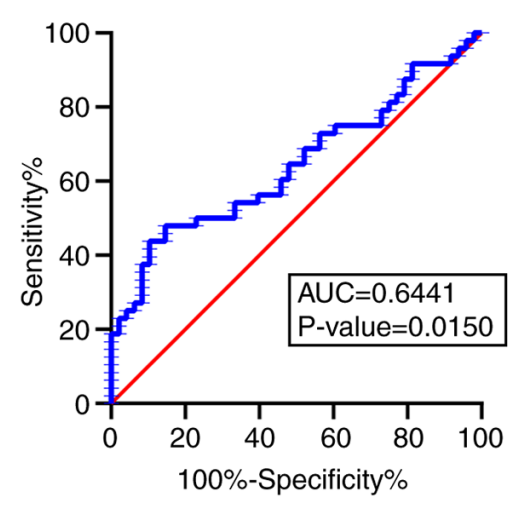

E

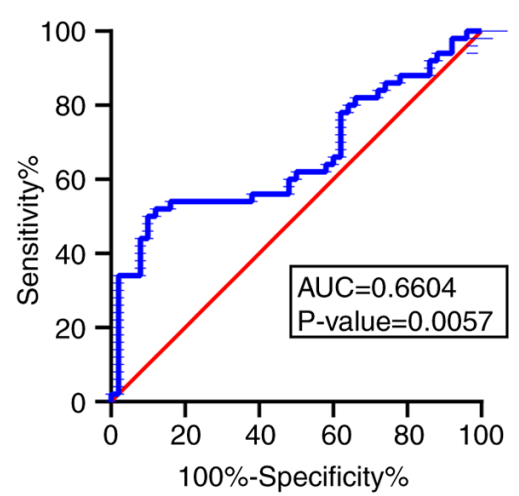

$\mathrm{H}$

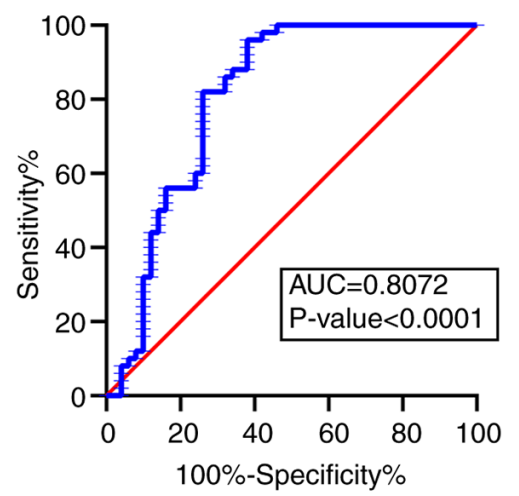

C

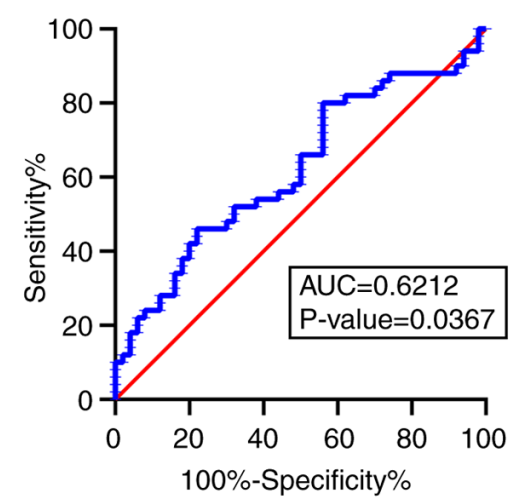

$\mathrm{F}$

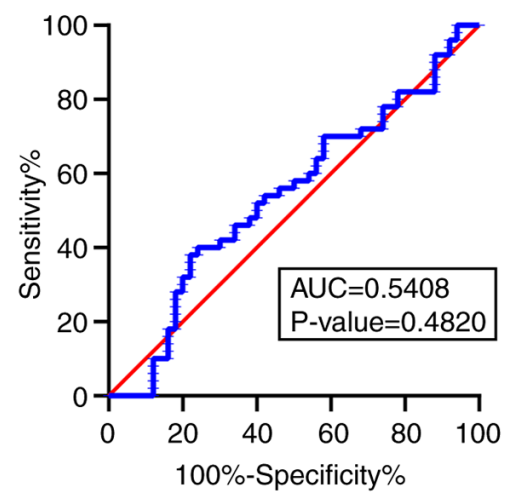

I

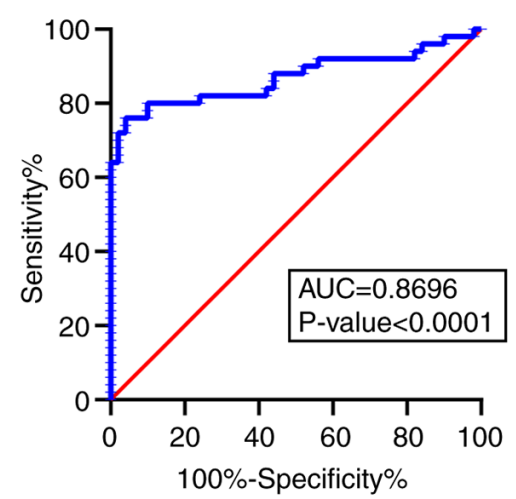

Figure 3. Receiver operating characteristic curves for mitochondrial dynamics- and biogenesis-associated mRNAs. AUC of (A) Mfn 1 is 0.6488 , of (B) Mfn 2 is 0.6441 , of (C) Opa 1 is 0.6212 , of (D) Drp 1 is 0.6468 , of (E) Fis 1 is 0.6604 , of (F) AMPK is 0.5408 , of (G) PGC-1 $\alpha$ is 0.8628 , of (H) TFAM is 0.8072 and of (I) NRF-1 is 0.8696 . Analysis based on the reverse transcription-quantitative PCR results in mRNAs plotted as sensitivity versus specificity. AUC $>0.5$ was considered significant. AUC, area under the curve; Mfn, mitofusion; Opa, optic atrophy; Drp, dynamin-related protein; Fis, fission; AMPK, AMP-activated protein kinase; PGC, peroxisome proliferator-activated receptor- $\gamma$ co-activator; NRF, nuclear respiratory factor; TFAM, mitochondrial transcription factor A. 
A
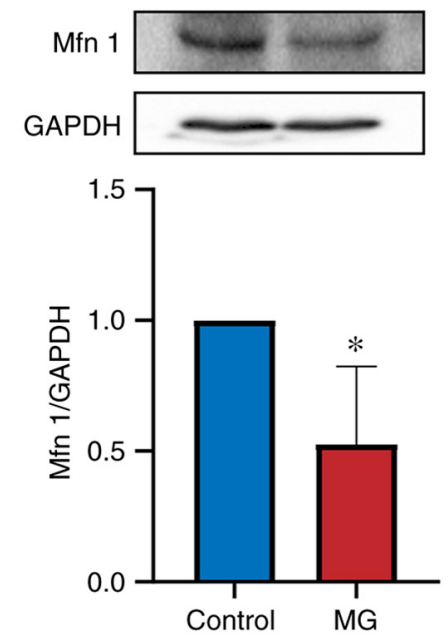

D
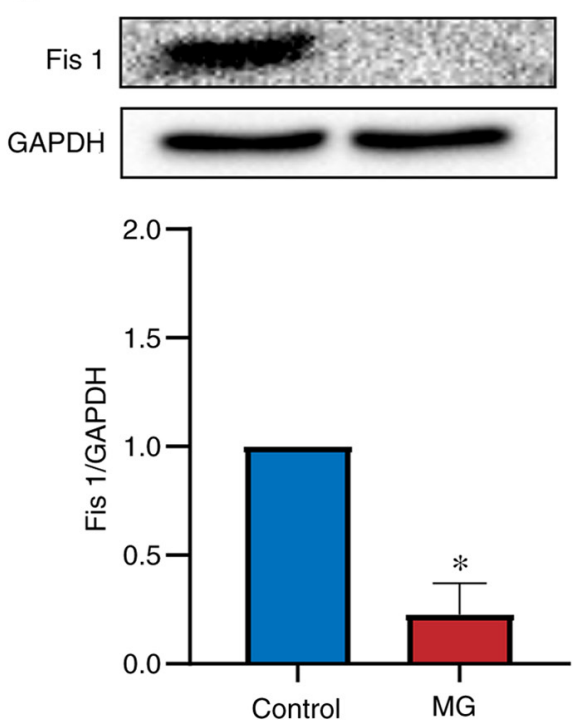

B
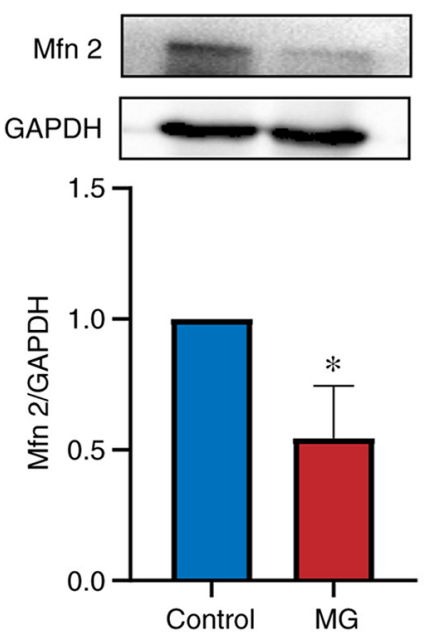

C
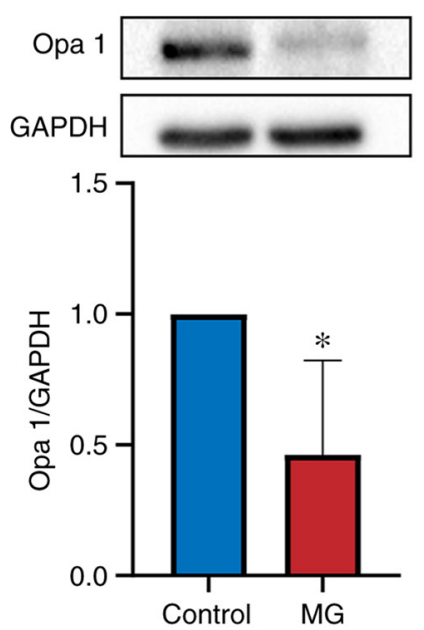

$E$
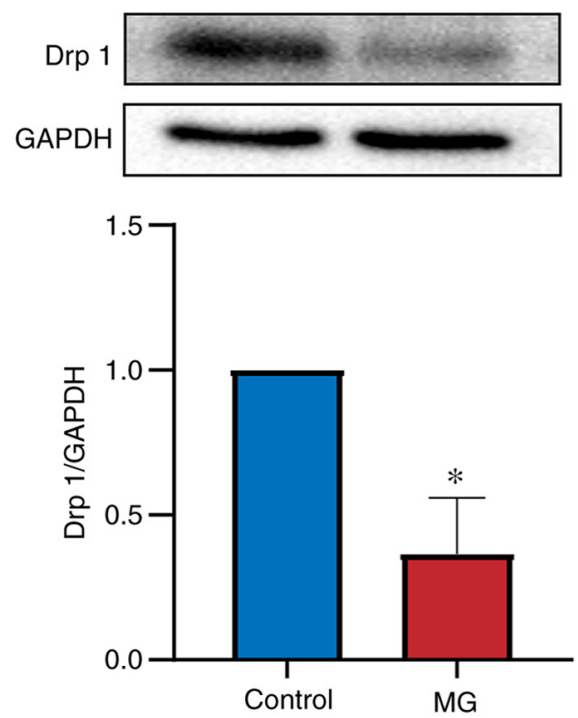

Figure 4. Levels of mitochondrial fusion/fission-associated protein in peripheral blood mononuclear cells. (A) Mfn1, (B) Mfn2, (C) Opa 1, (D) Fis 1 and (E) Drp 1 protein expression in control and MG group. Data are presented as the mean $\pm \mathrm{SD}$. ${ }^{*} \mathrm{P}<0.05$ vs. control. Mfn, mitofusion; Opa, optic atrophy; Drp, dynamin-related protein; Fis, fission; MG, myasthenia gravis.

of ATP hydrolysis, muscle myosin and actin perform muscle contraction by sliding on muscle fibers, leading to overall macro fiber shortening and densifying (31). Furthermore, studies $(32,33)$ have shown that muscle cells in patients with MG are highly sensitive to energy deficiency, which affects signal transduction and normal physiological activity of the neuromuscular junction. Histopathological analysis of extraocular muscle tissues of patients with MG has shown that myopathic features predominantly include substitution of muscle fibers by adipocytes and mitochondrial dysfunction at the ultrastructural level (34). Another study reported mitochondrial dysfunction in a patient with early-stage muscular dystrophy; these abnormal mitochondria were susceptible to further damage following sarcolemma injury (35). These results suggest that mitochondrial and neuromuscular disease are associated. Insufficient mitochondrial ATP synthesis leads to development of muscle movement disorders and clinical
MG symptoms, such as limb weakness, eyelid ptosis, chewing weakness, dysphagia and respiratory muscle paralysis (Fig. 6).

The most common method for MG diagnosis in clinical practice includes assessment of symptoms and signs of MG and a positive test for specific autoantibodies (36). However, a portion of patients with MG are not diagnosed as anti-AChR or anti-MuSK positive; thus, this method should be complemented.

By contrast with previous studies $(37,38)$ on $\mathrm{MG}$ that have concentrated on the immune system, the present study investigated mitochondrial dysfunction in muscles as a potential mechanism for MG. A large number of patients were needed to achieve reasonable results but the invasive nature of muscle tissue sampling causes pain and imposes a psychological burden on participants, resulting in reluctance to enroll in this type of study. This was a limitation of the present study. However, collecting blood samples for PBMC 
A
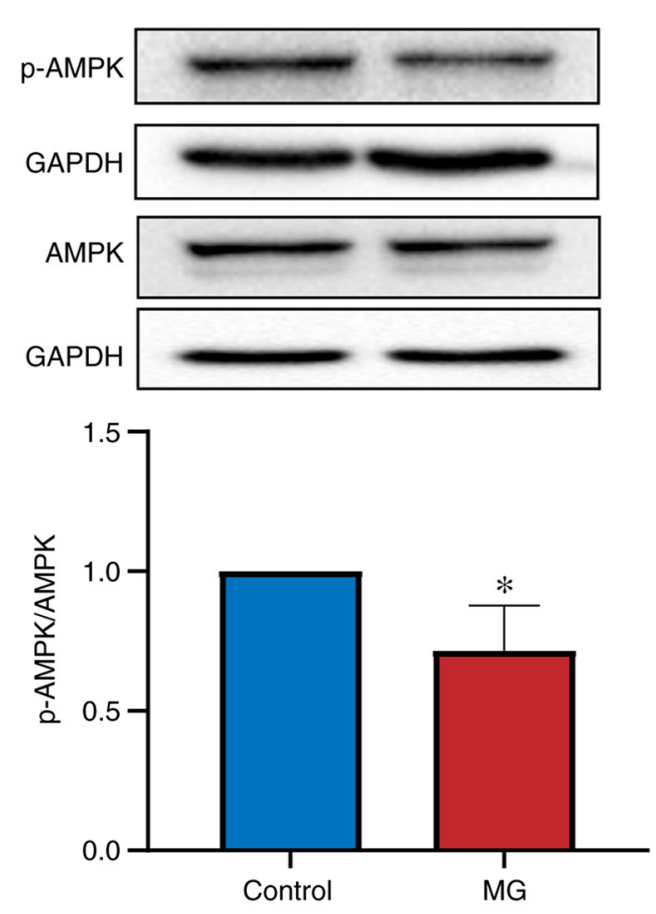

C
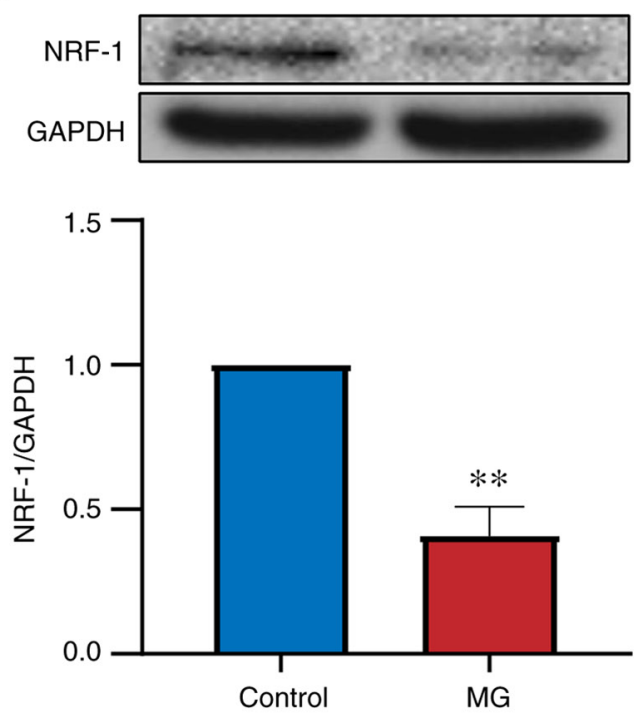

B
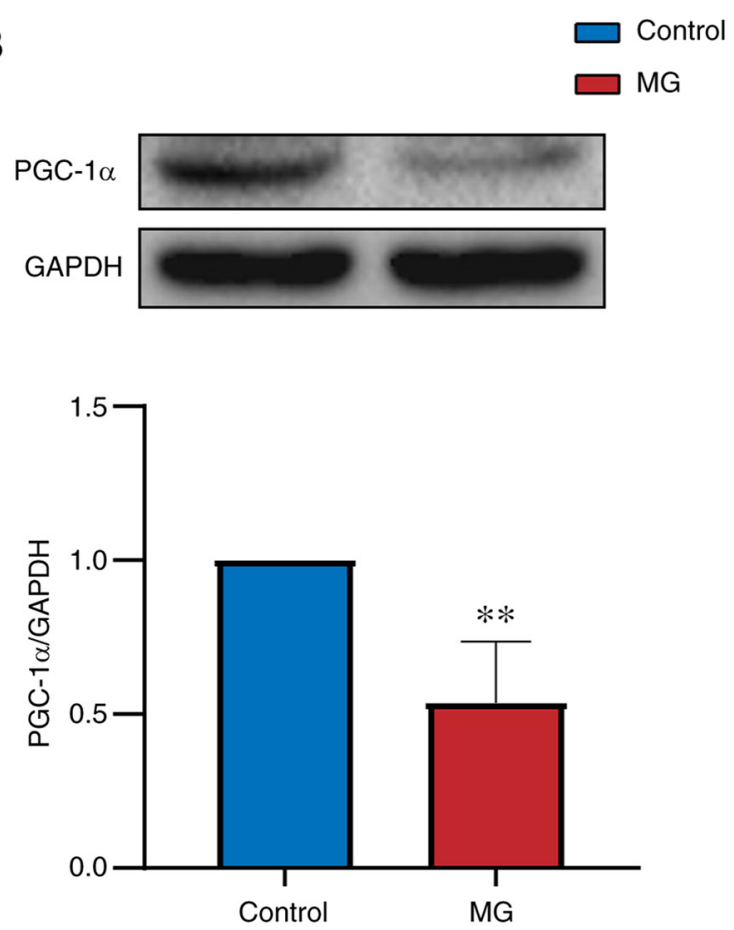

D
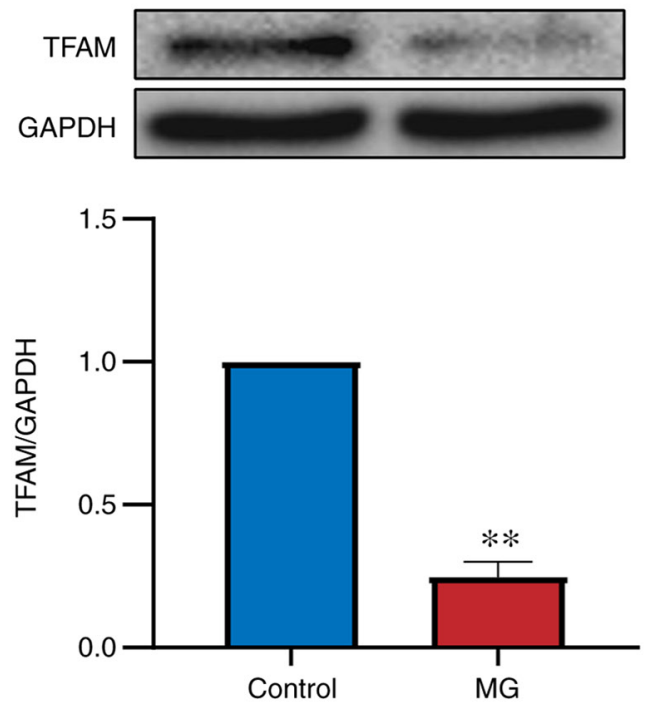

Figure 5. Expression levels of mitochondrial biosynthesis-associated protein in peripheral blood mononuclear cells. (A) AMPK, (B) PGC-1 $\alpha$, (C) NRF-1 and (D) TFAM protein expression levels in control and MG group. Data are presented as the mean \pm SD. ${ }^{*} \mathrm{P}<0.05$, ${ }^{* *} \mathrm{P}<0.01$ vs. control. AMPK, AMP-activated protein kinase; PGC, peroxisome proliferator-activated receptor- $\gamma$ co-activator; NRF, nuclear respiratory factor; TFAM, mitochondrial transcription factor A; p-, phosphorylated; MG, myasthenia gravis.

isolation is relatively painless, involves a simple procedure and has high patient compliance, which increases the feasibility of long-term research. PBMCs are used to identify cellular dysfunction associated with the pathophysiology of Parkinson's disease, a neurodegenerative disorder, such as decreased proteasome activity and mitochondrial dysfunction (39). Yalçınkaya et al (40) reported that gene expression analysis using PBMCs is a simple diagnostic method for Parkinson's disease. This research provided a reference for the present study to analyze the diagnostic value of MG by PBMCs.

Mitochondrial fission and fusion are key for immature cell proliferation as they provide cells with an adequate number of mature mitochondria for effective bioenergy genesis (41). Mitochondrial fusion helps mitochondria resist oxidative stress-induced damage (42). Studies have shown that mitochondrial fusion and fission impairment may affect mitochondrial function and lead to cardiomyocyte death $(43,44)$.

In the present study, mRNA and protein levels of fusionassociated genes Mfn1/2 and Opa1 were decreased in patients with MG compared with control subjects. Mfn1 promotes fusion of tethering-adjacent mitochondria in coordination with Opa1, whereas Mfn2 acts independently. Mitochondria of cardiomyocytes in Mfn-2-deficient mice are pleiomorphic, enlarged and exhibit functional deterioration $(15,45)$. 

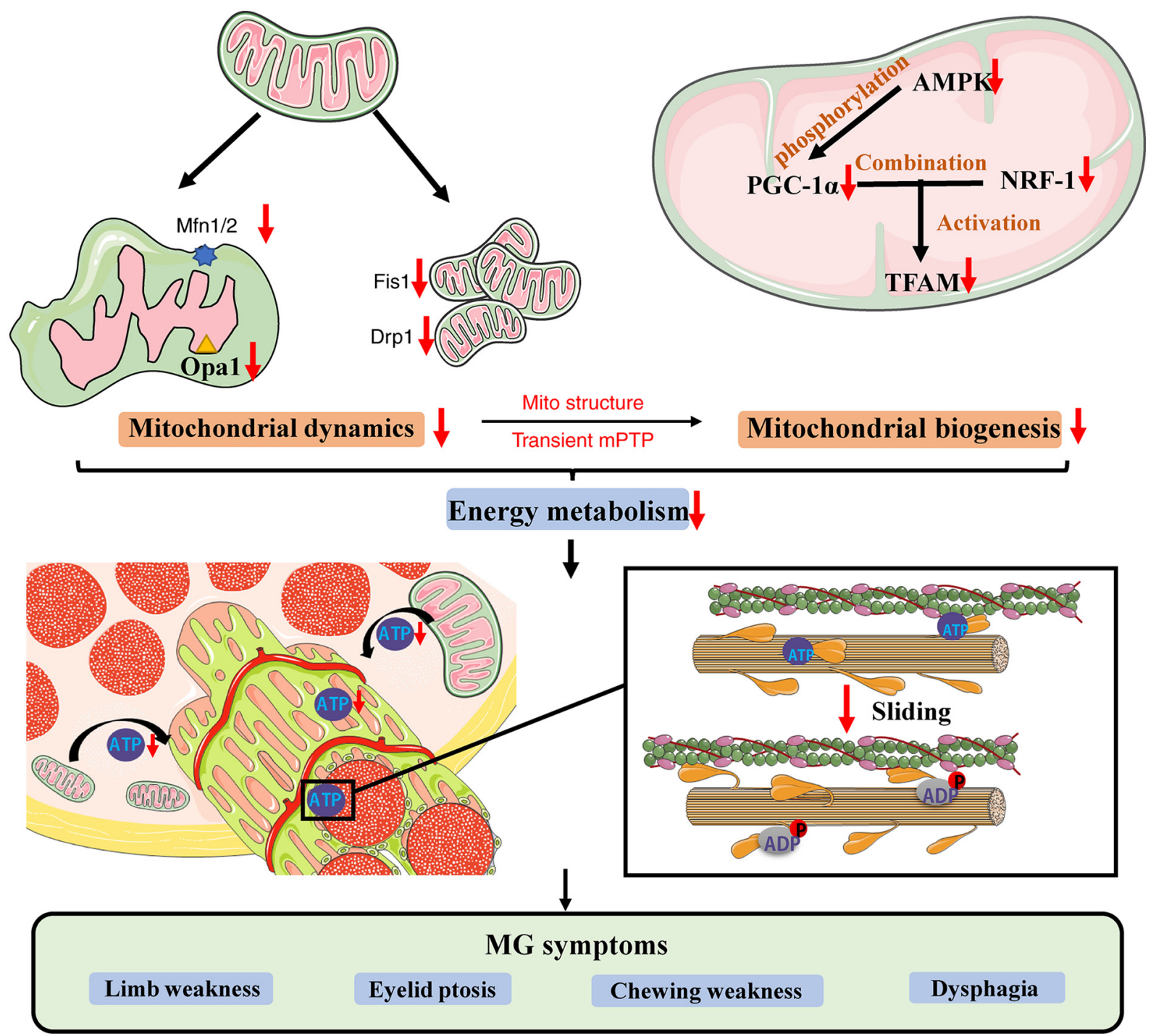

Figure 6. Schematic diagram of mitochondrial dynamics and biogenesis associated with MG. Decreased Mfn1/2, Opa1, Fis1 and Drp1 proteins leads to imbalance of mitochondrial fusion and fission, affecting mitochondrial structure and regulation of mitochondrial permeability transition pore channels. AMPK modulates activation of PGC-1 $\alpha$ and binding to NRF-1 to activate TFAM. Decreased expression of these proteins decreases mitochondrial biosynthesis, energy generation and ATP synthesis, affecting the binding of myosin and actin and causing MG symptoms, such as limb weakness, eyelid ptosis, chewing weakness and dysphagia. MG, myasthenia gravis; Mfn1/2, mitofusion1/2; Opa1, optic atrophy type 1; Drp1, dynamin-related protein 1; Fis1, fission 1; AMPK, AMP-activated protein kinase; PGC-1 $\alpha$, peroxisome proliferators activated receptor $\gamma$ coactivator $1 \alpha$; NRF-1, nuclear respiratory factor-1; TFAM, mitochondrial transcription factor A; mPTP, mitochondrial permeability transition pore.

Santel et al (46) found that the highest mRNA levels of Mfn1 and Mfn2 are present in energy-demanding tissue, such as skeletal muscle, heart and brain, which demonstrates the role of mitochondrial fusion in the energy supply chain.

Fission-associated gene products include Fis1 and Drp1. Drp1 translocates to the OMM following signaling from cytosolic GTPase and active fission sites (45). Fis1 recruits Drp1 to the mitochondria to regulate fission, which is associated with skeletal muscle mass. The impairment of mitochondrial fission has been shown to result in muscle atrophy $(8,47)$. These two proteins were expressed at lower levels in patients with MG than in control subjects. However, in the present study, mRNA expression levels of Drp1 and Fis1 were higher than expected compared with protein levels. Fis1 was barely detected in patients with MG, as confirmed by repeat testing. Therefore, it was hypothesized that other factors may have influenced the translation process to decrease protein synthesis.
Untranslated regions (UTRs) determine the fate of proteins by regulating their interactions. In most cases, single-stranded miRNAs are not fully complementary to the 3'-UTR of their target mRNA, thus blocking translation and regulating gene expression. Synthetic 5'-UTR RNA structures regulate protein translation in mammalian cells $(48,49)$. Circular RNAs also exhibit a potent translation regulatory function via their sponge function (50). These mechanisms only affect protein levels, not mRNA stability. Further investigation is required to determine the mechanism underlying differences in mRNA and protein expression levels.

Mitochondrial dynamics and biogenesis are reciprocally coupled. Mitochondrial fission-associated proteins induce opening of the mitochondrial permeability transition pore channel, which leads to changes in mitochondrial membrane potential and reactions in the mitochondrial respiratory chain (51), which enhance mitochondrial biogenesis. 
Mitochondrial biogenesis serves a vital role in metabolic health and plasticity. AMPK is an energy metabolism receptor that phosphorylates PGC-1 $\alpha$ and activates SIRT1 by increasing cellular NAD ${ }^{+}$levels. Furthermore, AMPK leads to increased expression of PGC-1 $\alpha$ (17). SIRT1 has been shown to interact with PGC-1 $\alpha$ to enhance mitochondrial biogenesis (52). The overexpression of PGC- $1 \alpha$ is an effective therapy for age-associated muscle loss. In addition, PGC- $1 \alpha$-deficient mice exhibit neurodegeneration, suggesting that PGC-1 $\alpha$ may be involved in the pathogenesis of neuromuscular disease $(53,54)$. PGC-1 $\alpha$ interacts with NRF-1, a member of the NRF-1 Cap'n'collar-Basic leucine zipper protein family of nuclear transcription factors, to increase TFAM expression and regulate mitochondrial biosynthesis. NRF-1 stimulates nuclear gene expression to promote mitochondrial respiratory reactions. TFAM is a member of the high-mobility-group-box domain-containing protein family that initiates transcription of mtDNA. Conditional knockout of TFAM in dopaminergic neurons in MitoPark mice results in decreased mtDNA levels $(55,56)$. Therefore, biosynthesis promotes mitochondrial ATP synthesis and increases the number of mitochondria to provide an energy reservoir for skeletal muscle contraction $(57,58)$.

Preliminary work by our group on the gastrocnemius muscle tissue of a rat model of autoimmune MG, in which mitochondria are vacuolated, showed that the cristae were broken, expression levels of fusion- and fission-associated proteins were decreased and $\mathrm{Na}^{+} / \mathrm{K}^{+}$-ATPase and $\mathrm{Ca}^{2+} / \mathrm{Mg}^{2+}$-ATPase activity was decreased compared with control rats $(59,60)$. ATPase activity was decreased to varying degrees in this rat model, leading to a decrease in ATP synthesis and inability of muscles to complete contraction and diastolic movement. Ke et al (33) suggested that the mitochondrial biogenesis signaling pathway is associated with MG, verifying the association between MG and mitochondrial dynamics and biogenesis.

In the present study, expression levels of fusion-associated proteins Mfn1/2 and Opa1 and fission-associated proteins Fis1 and Drp1 were significantly lower in patients with MG than in control subjects. However, the mRNA expression levels of Mfn1, Mfn2 and Opa1 decreased, while those of Fis1 and Drp1 increased in patients with MG compared with control subjects. Both the protein and mRNA expression levels of mitochondrial biogenesis-associated factors AMPK, PGC-1 $\alpha$, NRF-1 and TFAM were decreased in PBMCs of patients with MG. Of 50 patients with MG included in the present study, 6 patients were anti-AChR-negative. Gene expression analysis in these patients (anti-AChR-negative) demonstrated that mRNA expression level was consistent with the whole sample (including anti-AChR-negative and -positive) result, indicating that this subset of patients also have mitochondrial energy metabolism decreased.

ROC curve analysis showed that mitochondrial dynamics- and biogenesis-associated factors were specific and sensitive for diagnosing MG. Excluding AMPK, AUC values of the Mfn1/2, Opa1, Fis1, Drp1, PGC-1 $\alpha$, NRF-1 and TFAM were 0.6212-0.8696 ( $\mathrm{P}<0.05)$. Furthermore, the AUC values of PGC-1 $\alpha$, NRF-1 and TFAM were $>0.8$. A higher AUC value indicates a greater potential to distinguish patients from controls. Thus, ROC curve analysis indicated that these proteins may serve as promising biomarkers for MG. However, the present study had limitations. In the absence of clinical diagnosis, it is difficult to distinguish MG from other types of mitochondria-associated disease based on levels of mitochondrial indicators. In addition, the present study only collected patients with type IIb MG and obtained AUC $>0.8$, but this result is not comprehensive. The present study did not determine the explicit mechanism that how suffering MG underlying mitochondrial function disorder and to distinguish MG from other types of mitochondria-associated disease. In future, the potential diagnostic value of mitochondria-associated indicators in MG should be further researched.

In conclusion, expression levels of mitochondrial dynamics- and biogenesis-associated factors in PBMCs were significantly different between patients with MG and control subjects. These factors may serve as potential diagnostic biomarkers for MG.

\section{Acknowledgements}

Not applicable.

\section{Funding}

The present study was supported by National Natural Science Foundation of China (grant no. 81473568).

\section{Availability of data and materials}

The datasets used and/or analyzed during the current study are available from the corresponding author on reasonable request.

\section{Authors' contributions}

YS conceived and designed the experiments. LL, DC and $\mathrm{HZ}$ performed most of experiments and drafted the manuscript. JLiang, AJ and FL performed the formal analysis and interpretation of data. YS provided funding and gave final approval of the version to be published. JLi, ZC, QJ and PL carried out part of the PCR experiments and collected all clinical data. JS and WJ performed isolation of PBMCs and revised the manuscript from a critical perspective for important intellectual content. QL and LK performed the statistical analysis, confirmed the authenticity of all the raw data and gave final approval of the version to be published. JS and WJ agreed to be accountable for all aspects of the work in ensuring that questions related to the accuracy or integrity of any part of the work are appropriately investigated and resolved. All authors have read and approved the final manuscript.

\section{Ethics approval and consent to participate}

All participants understood the experimental procedure and provided written informed consent. Written informed consent was obtained from parents/guardians of all participants $<18$ years old. All procedures involving human subjects were approved by the Academic Ethics Committee of The First Affiliated Hospital of Guangzhou University of Chinese Medicine [approval no. ZYYZCK(2018)075]. 


\section{Patient consent for publication}

Not applicable.

\section{Competing interests}

The authors declare that they have no competing interests.

\section{References}

1. Binks S, Vincent A and Palace J: Myasthenia gravis: A clinical-immunological update. J Neurol 263: 826-834, 2016.

2. Berrih-Aknin S, Frenkian-Cuvelier M and Eymard B: Diagnostic and clinical classification of autoimmune myasthenia gravis J Autoimmun 48-49: 143-148, 2014.

3. Hehir MK and Silvestri NJ: Generalized myasthenia gravis: Classification, clinical presentation, natural history, and epidemiology. Neurol Clin 36: 253-260, 2018.

4. Sieb JP: Myasthenia gravis: An update for the clinician. Clin Exp Immunol 175: 408-418, 2014

5. Szczudlik P, Sobieszczuk E, Szyluk B, Lipowska M, Kubiszewska J and Kostera-Pruszczyk A: Determinants of quality of life in myasthenia gravis patients. Front Neurol 11 553626, 2020.

6. Mantegazza R and Cavalcante P: Diagnosis and treatment of myasthenia gravis. Curr Opin Rheumatol 31: 623-633, 2019.

7. Bastian TW, von Hohenberg WC, Georgieff MK and Lanier LM: Chronic energy depletion due to iron deficiency impairs dendritic mitochondrial motility during hippocampal neuron development J Neurosci 39: 802-813, 2019.

8. Romanello V and Sandri M: Mitochondrial quality control and muscle mass maintenance. Front Physiol 6: 422, 2016

9. Meyer JN, Leuthner TC and Luz AL: Mitochondrial fusion, fission, and mitochondrial toxicity. Toxicology 391: 42-53, 2017.

10. Fix DK, Hardee JP, Gao S, VanderVeen BN, Velázquez KT and Carson JA: Role of gp130 in basal and exercise-trained skeletal muscle mitochondrial quality control. J Appl Physiol (1985) 124: 1456-1470, 2018

11. Forrester SJ, Preston KJ, Cooper HA, Boyer MJ, Escoto KM Poltronetti AJ, Elliott KJ, Kuroda R, Miyao M, Sesaki H, et al: Mitochondrial fission mediates endothelial inflammation. Hypertension 76: 267-276, 2020.

12. Chiong M, Cartes-Saavedra B, Norambuena-Soto I, Mondaca-Ruff D, Morales PE, García-Miguel M and Mellado R: Mitochondrial metabolism and the control of vascular smooth muscle cell proliferation. Front Cell Dev Biol 2: 72, 2014.

13. Hu L, Ding M, Tang D, Gao E, Li C, Wang K, Qi B, Qiu J, Zhao H, Chang $\mathrm{P}$, et al: Targeting mitochondrial dynamics by regulating Mfn2 for therapeutic intervention in diabetic cardiomyopathy. Theranostics 9: 3687-3706, 2019.

14. Gao Q, Wang XM, Ye HW, Yu Y, Kang PF, Wang HJ, Guan SD and $\mathrm{Li} \mathrm{ZH}$ : Changes in the expression of cardiac mitofusin-2 in different stages of diabetes in rats. Mol Med Rep 6: 811-814, 2012.

15. Li Y and Liu X: Novel insights into the role of mitochondrial fusion and fission in cardiomyocyte apoptosis induced by ischemia/reperfusion. J Cell Physiol 233: 5589-5597, 2018.

16. Zhang M, Wu J, Sun R, Tao X, Wang X, Kang Q, Wang H, Zhang L, Liu P, Zhang J, et al: SIRT5 deficiency suppresses mitochondrial ATP production and promotes AMPK activation in response to energy stress. PLoS One 14: e 0211796, 2019.

17. Wen JJ, Cummins CB, Szczesny B and Radhakrishnan RS: Cardiac dysfunction after burn injury: Role of the AMPK-SIRT1-PGC1 $\alpha$-NFE2L2-ARE Pathway. J Am Coll Surg 230: 562-571, 2020.

18. Lv J, Bhatia M and Wang X: Roles of mitochondrial DNA in energy metabolism. Adv Exp Med Biol 1038: 71-83, 2017.

19. Zhao Q, Tian Z, Zhou G, Niu Q, Chen J, Li P, Dong L, Xia T, Zhang S and Wang A: SIRT1-dependent mitochondrial biogenesis supports therapeutic effects of resveratrol against neurodevelopment damage by fluoride. Theranostics 10: 4822-4838, 2020.

20. Araujo BG, Souza E, Silva LF, de Barros Torresi JL, Siena A Valerio BCO, Brito MD and Rosenstock TR: Decreased mitochondrial function, biogenesis, and degradation in peripheral blood mononuclear cells from amyotrophic lateral sclerosis patients as a potential tool for biomarker research. Mol Neurobiol 57: 5084-5102, 2020.
21. Santacatterina F, Chamorro M, de Arenas CN, Navarro C, Martín MA, Cuezva JM and Sánchez-Aragó M: Quantitative analysis of proteins of metabolism by reverse phase protein microarrays identifies potential biomarkers of rare neuromuscular diseases. J Transl Med 13: 65, 2015.

22. Oh KH, Sheoran S, Richmond JE and Kim H: Alcohol induces mitochondrial fragmentation and stress responses to maintain normal muscle function in Caenorhabditis elegans. FASEB J 34: 8204-8216, 2020

23. Sligar J, Debruin DA, Saner NJ, Philp AM and Philp A: The importance of mitochondrial quality control for maintaining skeletal muscle function across healthspan. Am J Physiol Cell Physiol: Feb 2, 2022 (Epub ahead of print). doi: 10.1152/ajpcell.00388.2021.

24. Sanders DB, Wolfe GI, Benatar M, Evoli A, Gilhus NE, Illa I, Kuntz N, Massey JM, Melms A, Murai H, et al: International consensus guidance for management of myasthenia gravis: Executive summary. Neurology 87: 419-425, 2016.

25. Livak KJ and Schmittgen TD: Analysis of relative gene expression data using real-time quantitative PCR and the 2(-Delta Delta $\mathrm{C}(\mathrm{T}))$ method. Methods 25: 402-408, 2001

26. Anil R, Kumar A, Alaparthi S, Sharma A, Nye JL, Roy B, O'Connor KC and Nowak RJ: Exploring outcomes and characteristics of myasthenia gravis: Rationale, aims and design of registry-The EXPLORE-MG registry. J Neurol Sci 414: 116830, 2020.

27. Park JS, Eah KY and Park JM: Epidemiological profile of myasthenia gravis in South Korea using the national health insurance database. Acta Neurol Scand: Feb 9, 2022 (Epub ahead of print). doi: 10.1111/ane.13596.

28. Zou M, Liu Z, Zhang XS and Wang Y: NCC-AUC: An AUC optimization method to identify multi-biomarker panel for cancer prognosis from genomic and clinical data. Bioinformatics 31 : 3330-3338, 2015 .

29. Zhang S, Ding JH, Zhou F, Wang ZY, Zhou XQ and Hu G: Iptakalim ameliorates MPP + -induced astrocyte mitochondrial dysfunction by increasing mitochondrial complex activity besides opening mitoK(ATP) channels. J Neurosci Res 87: 1230-1239, 2009

30. Shally A and McDonagh B: The redox environment and mitochondrial dysfunction in age-related skeletal muscle atrophy. Biogerontology 21: 461-473, 2020.

31. Powers JD, Malingen SA, Regnier M and Daniel TL: The sliding filament theory since Andrew Huxley: Multiscale and multidisciplinary muscle research. Annu Rev Biophys 50: 373-400, 2021.

32. Ruiter AM, Verschuuren JJGM and Tannemaat MR: Fatigue in patients with myasthenia gravis. A systematic review of the literature. Neuromuscul Disord 30: 631-639, 2020.

33. Ke L, Li Q, Song J, Jiao W, Ji A, Chen T, Pan H and Song Y: The mitochondrial biogenesis signaling pathway is a potential therapeutic target for myasthenia gravis via energy metabolism (Review). Exp Ther Med 22: 702, 2021.

34. Rautenbach RM, Pillay K, Murray ADN and Heckmann JM Extraocular muscle findings in myasthenia gravis associated treatment-resistant ophthalmoplegia. J Neuroophthalmol 37: 414-417, 2017.

35. Vila MC, Rayavarapu S, Hogarth MW, Van der Meulen JH, Horn A, Defour A, Takeda S, Brown KJ, Hathout Y, Nagaraju K and Jaiswal JK: Mitochondria mediate cell membrane repair and contribute to Duchenne muscular dystrophy. Cell Death Differ 24: 330-342, 2017

36. Gilhus NE: Myasthenia Gravis. N Engl J Med 375: 2570-2581, 2016.

37. Punga AR, Maddison P, Heckmann JM, Guptill JT and Evoli A: Epidemiology, diagnostics, and biomarkers of autoimmune neuromuscular junction disorders. Lancet Neurol 21: 176-188, 2022.

38. Smith VM, Nguyen H, Rumsey JW, Long CJ, Shuler ML and Hickman JJ: A Functional Human-on-a-Chip autoimmune disease model of myasthenia gravis for development of therapeutics. Front Cell Dev Biol 9: 745897, 2021.

39. White AJ, Wijeyekoon RS, Scott KM, Gunawardana NP, Hayat S, Solim IH, McMahon HT, Barker RA and Williams-Gray CH: The Peripheral Inflammatory Response to Alpha-Synuclein and Endotoxin in Parkinson's Disease. Front Neurol 9: 946, 2018.

40. Yalçınkaya N, Haytural H, Bilgiç B, Özdemir Ö, Hanağası H, Küçükali Cİ, Özbek Z, Akcan U, İdrisoğlu HA, Gürvit H and Tüzün E: Expression changes of genes associated with apoptosis and survival processes in Parkinson's disease. Neurosci Lett 615: 72-77, 2016 
41. Ding Q, Qi Y and Tsang SY: Mitochondrial biogenesis, mitochondrial dynamics, and mitophagy in the maturation of cardiomyocytes. Cells 10: 2463, 2021.

42. Ahmed ME, Selvakumar GP, Kempuraj D, Thangavel R, Mentor S, Dubova I, Raikwar SP, Zaheer S, Iyer S and Zaheer A Synergy in disruption of mitochondrial dynamics by $\mathrm{A} \beta$ (1-42) and glia maturation factor (GMF) in SH-SY5Y cells is mediated through alterations in fission and fusion proteins. Mol Neurobiol 56: 6964-6975, 2019.

43. Gao J, Zhao L, Wang J, Zhang L, Zhou D, Qu J, Wang H, Yin M, Hong $\mathrm{J}$ and Zhao W: C-Phycocyanin ameliorates mitochondrial fission and fusion dynamics in ischemic cardiomyocyte damage. Front Pharmacol 10: 733, 2019.

44. Zhang Q, Guo D, Wang Y, Wang X, Wang Q, Wu Y, Li C, Wang W and Wang Y: Danqi pill protects against heart failure post-acute myocardial infarction via HIF- $\alpha /$ PGC-1 $\alpha$ mediated glucose metabolism pathway. Front Pharmacol 11: 458, 2020.

45. Iqbal S and Hood DA: The role of mitochondrial fusion and fission in skeletal muscle function and dysfunction. Front Biosci (Landmark Ed) 20: 157-172, 2015.

46. Santel A, Frank S, Gaume B, Herrler M, Youle RJ and Fuller MT: Mitofusin-1 protein is a generally expressed mediator of mitochondrial fusion in mammalian cells. J Cell Sci 116: 2763-2774, 2003.

47. Fix DK, VanderVeen BN, Counts BR and Carson JA: Regulation of skeletal muscle DRP-1 and FIS-1 protein expression by IL-6 signaling. Oxid Med Cell Longev 2019: 8908457, 2019.

48. Eisenhut P, Mebrahtu A, Moradi Barzadd M, Thalén N Klanert G, Weinguny M, Sandegren A, Su C, Hatton D, Borth N and Rockberg J: Systematic use of synthetic 5'-UTR RNA structures to tune protein translation improves yield and quality of complex proteins in mammalian cell factories. Nucleic Acids Res 48: e119, 2020.

49. Mayr C: Regulation by 3'-Untranslated Regions. Annu Rev Genet 51: 171-194, 2017.

50. Prats AC, David F, Diallo LH, Roussel E, Tatin F, Garmy-Susini B and Lacazette E: Circular RNA, the key for translation. Int J Mol Sci 21: 8591, 2020.

51. Cortassa S, Aon MA, Winslow RL and O'Rourke B: A mitochondrial oscillator dependent on reactive oxygen species. Biophys J 87: 2060-2073, 2004.
52. Reutzel M, Grewal R, Dilberger B, Silaidos C, Joppe A and Eckert GP: Cerebral mitochondrial function and cognitive performance during aging: A longitudinal study in NMRI mice. Oxid Med Cell Longev 2020: 4060769, 2020

53. Xiang Z, Valenza M, Cui L, Leoni V, Jeong HK, Brilli E, Zhang J, Peng Q, Duan W, Reeves SA, et al: Peroxisome-proliferator-activated receptor gamma coactivator $1 \alpha$ contributes to dysmyelination in experimental models of Huntington's disease. J Neurosci 31: 9544-9553, 2011.

54. Jones AW, Yao Z, Vicencio JM, Karkucinska-Wieckowska A and Szabadkai G: PGC-1 family coactivators and cell fate: Roles in cancer, neurodegeneration, cardiovascular disease and retrograde mitochondria-nucleus signalling. Mitochondrion 12: 86-99, 2012.

55. Martín-Jiménez R, Lurette $\mathrm{O}$ and Hebert-Chatelain E: Damage in mitochondrial DNA associated with Parkinson's Disease. DNA Cell Biol 39: 1421-1430, 2020.

56. Islam H, Bonafiglia JT, Turnbull PC, Simpson CA, Perry CGR and Gurd BJ: The impact of acute and chronic exercise on Nrf2 expression in relation to markers of mitochondrial biogenesis in human skeletal muscle. Eur J Appl Physiol 120: 149-160, 2020.

57. Piao Y, Kim HG, Oh MS and Pak YK: Overexpression of TFAM, NRF-1 and myr-AKT protects the MPP(+)-induced mitochondrial dysfunctions in neuronal cells. Biochim Biophys Acta 1820: 577-585, 2012.

58. Ramachandran B, Yu G and Gulick T: Nuclear respiratory factor 1 controls myocyte enhancer factor $2 \mathrm{~A}$ transcription to provide a mechanism for coordinate expression of respiratory chain subunits. J Biol Chem 283: 11935-11946, 2008.

59. Song J, Lei X, Jiao W, Song Y, Chen W, Li J and Chen Z: Effect of Qiangji Jianli decoction on mitochondrial respiratory chain activity and expression of mitochondrial fusion and fission proteins in myasthenia gravis rats. Sci Rep 8: 8623, 2018

60. Jiao W, Hu F, Li J, Song J, Liang J, Li L, Song Y, Chen Z, Li Q and Ke L: Qiangji Jianli Decoction promotes mitochondrial biogenesis in skeletal muscle of myasthenia gravis rats via AMPK/PGC-1 $\alpha$ signaling pathway. Biomed Pharmacother 129: 110482, 2020.

(7) $\Theta$ This work is licensed under a Creative Commons

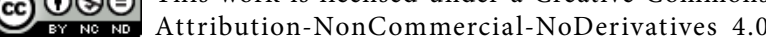
International (CC BY-NC-ND 4.0) License. 